This item was submitted to Loughborough's Research Repository by the author.

Items in Figshare are protected by copyright, with all rights reserved, unless otherwise indicated.

\title{
Density-functional study of adsorption of Co on $\mathrm{Si}(100)$
}

\section{PLEASE CITE THE PUBLISHED VERSION}

http://dx.doi.org/10.1103/PhysRevB.64.245332

\section{PUBLISHER}

(C) American Physical Society

\section{VERSION}

AM (Accepted Manuscript)

\section{PUBLISHER STATEMENT}

This work is made available according to the conditions of the Creative Commons Attribution-NonCommercialNoDerivatives 4.0 International (CC BY-NC-ND 4.0) licence. Full details of this licence are available at: https://creativecommons.org/licenses/by-nc-nd/4.0/

\section{LICENCE}

CC BY-NC-ND 4.0

\section{REPOSITORY RECORD}

Horsfield, Andrew P., Steven D. Kenny, and Hideaki Fujitani. 2015. "Density-functional Study of Adsorption of Co on Si(100)". figshare. https://hdl.handle.net/2134/19685. 


\title{
A density functional study of adsorption of Co on $\operatorname{Si}(100)$
}

\author{
Andrew P. Horsfield \\ fecit, 2 Longwalk Road, Stockley Park, Uxbridge, Middlesex UB11 1AB \\ Steven D. Kenny \\ Department of Mathematical Sciences, Loughborough University, Loughborough, Leicestershire \\ LE11 3TU
}

Hideaki Fujitani

Fujitsu Laboratories Ltd., 10-1 Morinosato-Wakamiya, Atsugi 243-01, Japan 


\begin{abstract}
We have studied the stable sites for Co both on the surface of $\operatorname{Si}(100)$ and subsurface using ab initio methods. We show that the most stable surface site for Co is situated in the dimer trenches (the low site). The subsurface sites that we study are all found to be more stable than the most stable surface site. The most stable subsurface site is the under dimer site. The most stable site of all is, however, the dimer vacancy site formed by removing the dimer above the cobalt in the under dimer site.
\end{abstract}

\title{
I. INTRODUCTION
}

The central semiconductor technology used to produce the very large scale integrated (VLSI) circuits that form the core of personal computers and consumer electronics is the complementary metal oxide semiconductor (CMOS) transistor. CMOS transistors are constructed on silicon wafers by a combination of implantation of dopants to produce active regions (source, drain and channel), the growth of surface oxide (to insulate regions from one another), and metalization to form contacts to, and wires between, the devices.

A number of materials have been used to form the metallic contacts. One very popular choice is $\mathrm{TiSi}_{2}$. However, as devices have become smaller, its limitations have become apparent. The high resistivity phase (C49) has a lower surface energy, but a higher formation energy, than the low resistivity phase (C54) [1]. Thus the C49 phase nucleates and grows on the silicon surface, but transforms to the C54 phase after a high temperature anneal ( $\mathrm{T}$ $>700^{\circ} \mathrm{C}$ ). However, for very small devices, for which there is a large surface area to volume ratio, the transformation requires higher temperatures. This makes $\mathrm{TiSi}_{2}$ unattractive.

Thus a new material has been sought, and $\mathrm{CoSi}_{2}$ has now become popular because of its desirable properties: a very low resistivity $(14-17 \mu \Omega \mathrm{cm})[2]$; the anneal needed to bring about the phase transition from CoSi to $\mathrm{CoSi}_{2}$ after growth occurs at a low temperature (around 


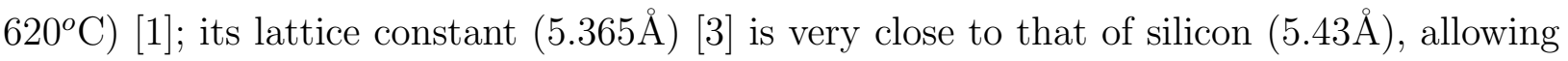
for defect free epitaxial growth; because it is a silicide, growth from previously deposited silicon ensures that the gate contact is properly aligned. However, $\mathrm{CoSi}_{2}$ introduces some new problems, notably that the structures that grow are sensitive to the surface structure of the silicon substrate [4].

In this work we wish to understand how the first fraction of a monolayer of cobalt binds to a clean (100) silicon surface. The early stages of the reaction of Co with Si will define the environment in which subsequent Co atoms will interact with the Si substrate. Thus a thorough understanding of the very early stages is important to our general understanding of the formation of cobalt silicides on silicon. In particular we wish to know what the preferred sites for adsorption are on the perfect $(2 \times 2)$ buckled dimer surface, and what effect the adsorption has on the surface reconstruction.

Below we summarize some relevant experimental findings. We then describe the computational methods that we used, and present the results that we found. We conclude with some comments about the implications of what we find.

\section{EXPERIMENTAL BACKGROUND}

The scanning tunneling microscopy experiments of Scheuch et al. [7] provide detailed structural information from 0.01 monolayer coverage up to 30 monolayer coverage on smooth $\mathrm{Si}(100)$ surfaces. We are interested in the results for submonolayer coverages. At very low coverages they find that the dimer rows reorder, forming ribbons running perpendicular to the dimer rows, separated by missing dimer rows. The ribbons are about $30 \AA$ wide, and are formed from quasi-periodic $(2 \times n)$ structures with $n$ between 6 and 9 . This structure can be understood in terms of elasticity theory [8], though there remains the question of what exactly the role of the Co atoms is. At coverages of about 0.1 monolayers, two dimensional islands form that are one atomic plane high, which run perpendicular to the silicon dimer rows, but whose composition is unclear. 
The extended x-ray absorption fine structure measurements of Meyerheim et al. [4] show that the adsorption of Co onto Si depends strongly on the surface structure. The (100) surface of $\mathrm{Si}$ is prepared in two ways: by chemical etching followed by annealing, and by sputtering followed by annealing. On the chemically etched (smoother) surface it is found that the adsorbed Co lies in the plane of an unreconstructed $\operatorname{Si}(100)$ with about 2 nearest neighbor and 5 second neighbor Si atoms. On the sputtered (rougher) surface, the Co has around 8 Si neighbors, characteristic of $\mathrm{CoSi}_{2}$.

Cho et al [9] used coaxial impact collision ion scattering spectroscopy (CAICISS) to study the atomic structure of $\mathrm{Co}$ on $\mathrm{Si}(001)$ for coverages of $0.6 \mathrm{ml}$ to $1.9 \mathrm{ml}$. Their analysis caused them to conclude that the preferred adsorbtion sites are on top of a Si dimer (our D site - see below) and spanning the trench (our T3 site - see below).

\section{METHODS}

All the calculations are based on the Kohn-Sham formulation of the density functional theory of the electron gas [10]. The basis set used is a linear combination of atomic type orbitals (LCAO). The calculations were performed using the program Plato [11]. We work at the level of the local density approximation, using the functional of Goedecker et al. [12], and the relativistic pseudopotentials of Hartwigsen et al. [13]. Two basis sets are used: minimal and double numeric with polarization (DNP) [11]. The minimal basis set is used to carry out a rapid exploration of potentially interesting structures, and the larger basis set is used to refine the calculations to obtain more accurate geometries and energies. Unless otherwise stated, all geometries and energies reported in this paper are from double numeric basis set calculations.

To optimize the DNP basis set, we adjusted the cutoff radius. Both surface and bulk systems need to be considered. In Table I are given results of the optimization for bulk silicon, and a (001) surface of silicon. For the bulk an 8 atom cell was used, with a $6 \times 6 \times 6$ mesh of $\mathrm{k}$ points. A slab geometry with two equivalent surfaces was used for the surface 
calculation. A $2 \times 1$ surface unit cell with 8 layers of silicon, and a $4 \times 8 \times 1 \mathrm{k}$ point sampling, was used. From Table I we see that a cutoff of $r_{c}=8$ bohr radii is optimal. To get significantly better results we need to go to a triple numeric basis set. The plane wave calculations were performed using the same pseudopotentials and exchange and correlation functional as for the LCAO calculations. The program ABINIT [14] was used for the plane wave calculations.

As a further indication of the accuracy of the basis sets we looked at the binding energy curve for bulk $\mathrm{CoSi}_{2}$ and compared the results with well converged full potential, linearised augmented plane wave (FLAPW) calculations, plane wave pseudopotential calculations, and experiment. From Table II we see that the larger LCAO basis set is in very good agreement with the FLAPW calculation, and that even the minimal basis set result is quite respectable. Closer inspection of the minimal basis set results shows, however, that it is not adequate to describe accurately the charge distribution in the bonds in cobalt silicides.

\section{RESULTS AND DISCUSSION}

\section{A. The clean $\mathrm{Si}(100)$ surface}

We are interested in the addition of a submonolayer coverage of Co on the (100) surface of Si. Thus first we must look at the clean surface. We set up the clean $\operatorname{Si}(100)$ surface using a slab geometry with one surface terminated with hydrogen to saturate dangling bonds. The other surface was relaxed to form a $2 \times 2$ buckled dimer surface. The atoms in the two layers of Si neighboring the $\mathrm{H}$ atoms were frozen, with their positions corresponding to the experimental lattice constant of $5.43 \AA$. The $\mathrm{H}$ atoms and the remaining six layers of $\mathrm{Si}$ atoms were free to relax. Each layer has eight Si atoms in a c $(4 \times 4)$ unit cell, giving a total of 80 atoms in the unit cell (64 silicon and 16 hydrogen atoms). The cell was setup such that there was a seperation of $11 \AA$ between the Si surface and the terminating hydrogens. A k pointmesh of $4 \times 4 \times 1$ points (reduced to a total of 8 points by time reversal symmetry) 
was used. The bond length between the surface dimer atoms was $2.29 \AA$, and the tilt angle was $18.9^{\circ}$. These compare favorably with other similar calculations: $2.33 \AA$ and $18.0^{\circ}[16]$; $2.34 \AA$ and $16.5^{\circ}[17]$.

\section{B. Adsorption of one Co atom on $\mathrm{Si}(100)$}

We looked at the adsorption of a single Co atom on the $\operatorname{Si}(100) 2 \times 2$ reconstructed surface to see where Co is most likely to reside after deposition. Eleven sites were considered: the asymmetric pedestal site, $\mathrm{P}_{a}$; the symmetric pedestal site, $\mathrm{P}_{s}$; two trench sites, T3 and T4; the high site, H; the low site, L; the dimer site, D; the dimer vacancy site, DV; the site under the dimer, $\mathrm{U}_{D}$; the site under the high site, $\mathrm{U}_{H}$; and the site under the low site, $\mathrm{U}_{L}$. These are shown in Figure 1. For the first seven of these sites a Co atom is placed directly on the $\mathrm{Si}(100)$ surface. For Co to occupy the eighth site (DV), a Si dimer is first removed, and the Co atom then placed at this site. The remaining three sites $\left(\mathrm{U}_{D}, \mathrm{U}_{H}, \mathrm{U}_{L}\right)$ are all subsurface sites. Note that for the relaxation calculations of Co at all these sites, the same unit cell and $\mathrm{k}$-point sampling is used as for the clean surface. The $\mathrm{H}$ layer and the neighboring two $\mathrm{Si}$ layers are taken from the calculation of the clean $\operatorname{Si}(100)$ surface, and their positions frozen.

The $\mathrm{DV}$ and $\mathrm{U}_{D}$ structures were motivated by calculations performed for Ti adsorption on Si $[17,18]$. Miwa and Fukumoto [17] found that the vacant dimer site was energetically highly favourable for $\mathrm{Ti}(0.6 \mathrm{eV}$ more so than the pedestal site). Yu et al [18] found that the interstitial site, $\mathrm{U}_{D}$, was energetically favorable, and further was an important intermediate structure for the ejection of a Si dimer leading to the formation of the Ti-in-dimer-vacancy structure.

The $\mathrm{P}_{s}, \mathrm{~L}, \mathrm{H}, \mathrm{U}_{D}, \mathrm{U}_{L}$ and $U_{H}$ sites were found to be important in the reaction of $\mathrm{Ni}$ with Si in the absence of hydrogen termination by Higai and Ohno [16]. In particular they found that the penetration of $\mathrm{Ni}$ below the surface generally reduces the energy.

The D, T3 and T4 sites are important for a comparison with the experimental work of Cho et al [9]. 
The DV site has two Si atoms fewer than the others, so to compare energies, we need to account for the missing atoms. If we assume that the Co-on-dimer-vacancy structure forms by ejecting two Si atoms which then join the Si bulk elsewhere, then the energy we require for making comparisons is $E(\mathrm{Co}-$ on - dimer - vacancy $)=E_{D V}+2 E_{S i}$, where $E_{D V}$ is the energy found using the unit cell for the structure with the Co atom in the dimer vacancy site, and $E_{S i}$ is the energy per atom in bulk silicon.

The final relaxed geometries are shown in Figure 2, and the relative energies are given in Table III (the symmetric pedestal site is taken as the zero energy site). Of the seven simple adsorption sites $\left(\mathrm{P}_{s}, \mathrm{P}_{a}, \mathrm{~T} 3, \mathrm{~T} 4, \mathrm{H}, \mathrm{L}, \mathrm{D}\right)$, site $\mathrm{L}$ is the most stable by $0.24 \mathrm{eV}$ (equivalent to about 2800K). Interestingly, the three highest energy sites are D, T4 and T3, two of which (D and T3) are important sites according to Cho et al [9]. This suggests either that there are large energy barriers for escape from these sites (they are stabilised for kinetic reasons), or there are alternative interpretations of the experimental data. The DV and sub-surface sites are very low in energy, consistent with the results for $\mathrm{Ni}$ and $\mathrm{Ti}$. However, the amount of stabilisation is rather greater for Co.

In Table III is included a second value (in parenthesis) for the $\mathrm{U}_{D}$ value for Co. This value was found from a well converged plane wave calculation (the cutoff energy for the basis set was $340 \mathrm{eV}$ ). The code CASTEP [24] was used with the geometries of the reference structure $\left(\mathrm{P}_{s}\right)$ and the $\mathrm{U}_{D}$ structure taken from the Plato calculations (though with slightly different geometries from those used for the final results here). The good agreement between the two methods confirms the reliability of the atomic orbital type basis set for surface calculations.

We have found that two simple rules help explain the ordering of the energetics of these structures. The rules are:

1. Co prefers sites with high coordinations, with shorter bonds being stronger than longer ones

2. Co bonds to surface Si dimer atoms are strengthened because of the reduced coordination of the $\mathrm{Si}$ 
It is generally the case that short bonds are stronger than long ones since this leads to the lowering of the energy of bonding orbitals. So the only point in the first rule that needs explaining is the ability of Co to form many bonds. Co has a partially filled d shell and a low lying empty p shell into which valence s and d electrons can be easily promoted. This provides considerable flexibility when forming bonds. The preference of Co for high coordination can be seen by the fact that it forms eight covalent bonds in $\mathrm{CoSi}_{2}$. Thus the limiting factor in its ability to form bonds will be geometry, as this will determine how many Si atoms a Co atom can get close to.

Note that the strength of the bond between atoms $i$ and $j\left(U_{i j}\right)$ is measured in this analysis by the expression $U_{i j}=\sum_{\alpha \beta} H_{i \alpha, j \beta} \rho_{j \beta, i \alpha}$, where $\alpha$ and $\beta$ are atomic orbital indices, $H_{i \alpha, j \beta}$ is the Kohn-Sham Hamiltonian matrix, and $\rho_{j \beta, i \alpha}$ is the density matrix.

The second rule is to be expected since $\mathrm{Si}$ atoms that form the surface dimers are under coordinated, having charge in dangling bonds. Thus it is energetically favorable for these Si atoms to form additional bonds, with the charge in the dangling bonds contributing to the new bond charge.

Given these rules it is clear why the silicon on the dimer (D) site is the most weakly bound state: the Co atom at this site can only form two bonds to Si atoms. The individual bonds formed in this case are, however, very strong due to the fact that both the Si atoms bonded to originally had dangling bonds. When the Co bonds to this site it causes the Si dimer to flatten as can be seen in Figure 2 D. Similarly the Co atom in the two trench sites T3 and T4 (Figure 2 T3 and T4), is relatively weakly bound. In this case the Co atom forms more bonds (six) but because the local structure is open in these sites the Co atom is unable to form strong bonds with all its neighbours.

There are two possible Co positions on the pedestal site (Figure $2 \mathrm{P}_{s}$ and $\mathrm{P}_{a}$ ), which we have labelled the symmetric $\left(\mathrm{P}_{s}\right)$ and assymmetric $\left(\mathrm{P}_{a}\right)$ pedestal sites. The symmetric pedestal site is formed when the Co atom sits in the centre between the two dimers whilst in the asymmetric case the Co moves towards one end of the dimers. The bonding in these two sites is very similar with the Co in both sites forming six bonds to surrounding Si atoms, 
four strong bonds are formed with the four dimer atoms with two weaker bonds to the atoms in the second layer. The $\mathrm{P}_{s}$ site is slightly more stable because is it able to form four almost identical bonds to the atoms in the two surrounding dimers wheras the $\mathrm{P}_{a}$ site has one bond which is appreciably weaker.

The high (H) and low (L) sites (Figure $2 \mathrm{H}$ and L) are both very stable as they allow the Co atom to move into a relatively closed packed environment and form six bonds to surrounding $\mathrm{Si}$ atoms, one of which is an under-coordinated dimer atom. The high site is the higher energy site because the Co atom forms a weaker bond with the dimer atom than is the case in the low site. This is due to the Co-Si bond distance being greater. The Co-Si bond in the high site also weakens the dimer bond, stretching it from $2.29 \AA$ to $2.39 \AA$.

The under high $\left(\mathrm{U}_{H}\right)$ site (Figure $2 \mathrm{U}_{H}$ ), and under low $\left(\mathrm{U}_{L}\right)$ site (Figure $2 \mathrm{U}_{L}$ ), sites are very close in energy. In these sites the Co atom forms a large number of relatively weak bonds with surrounding Si atoms. Thus both of these sites are more stable than any of the surface sites for Co. The under dimer site (Figure $2 \mathrm{U}_{D}$ ) is more stable again due to the fact that as well as sitting in a close-packed environment the Co atom also forms strong bonds as it is able to bond to the under coordinated surface Si atoms. This is consistent with the findings of Bennett et al [25] who found that at low coverages Co preferentially occupies sites directly under three-fold coordinated surface $\mathrm{Si}$ atoms on the $\mathrm{Si}(111)$ surface.

The Co atom in the dimer vacancy site also sits in a close-packed environment. In this case four of the Si atoms bonding to the Co atom are under coordinated which allows the formation of strong bonds between it and the Si atoms. The other factor which makes this site more stable than the under dimer site is that in the under dimer case the dimer bond is substantially weakened by the Co atom. This introduces an energy penalty for Co forming bonds to its Si neighbors.

The stability of the DV site is consistent with the experimental findings of Meyerheim et al [4]. They observe that for the wet chemical etched (smooth) surface the Co atoms lie in the surface of the Si, with four Si neighbors in the plane, and two closer neighbors in the plane below. This is the DV site. For the argon sputtered surface they observe the formation 
of $\mathrm{CoSi}_{2}$. Presumably the surface has been partially amorphised by the sputtering. This has two consequences: there will be a higher concentration of under coordinated Si atoms with which Co can form strong bonds; the surface Si atoms will be able to reorient themselves locally more easily, thus easing the formation of a new phase.

In Table III are also given previously published values for $\mathrm{Ti}$ and $\mathrm{Ni}$ on $\mathrm{Si}(001)$. The general observation is that Co benefits more from being surrounded by Si than do either $\mathrm{Ti}$ or Ni. Thus the energies for Co with few Si neighbors are higher than the corresponding values for the other metals (see, for example, the T4 site), while those for Co with more Si neighbors are lower (see, for example, the $\mathrm{U}_{D}$ site).

\section{CONCLUSIONS}

In the present work possible bonding sites for a Co atom on a $\mathrm{Si}(100)$ surface have been studied. It has been shown that the Co atom likes to sit in a high coordination site preferably bonding to under coordinated $\mathrm{Si}$ atoms. This mean that the most stable surface site is the low site (L). In this site the Co atom forms six bonds to surrounding Si atoms, one of which is an under coordinated dimer atom. Due to the desire of the Co atom to sit in a site with a high coordination the subsurface sites are all more stable, as these sites allow the Co atoms to sit in a close packed environment. The under dimer site is the most stable of these due to the ability of the Co atom to bond to the under coordinated Si atoms that form the dimers. The dimer vacancy is the most stable site of all as it achieves the best compromise between coordination and bonding to Si atoms which are under coordinated. Finally we note that the most important difference between $\mathrm{Co}$ and $\mathrm{Ti}$ or $\mathrm{Ni}$ is that Co benefits considerably more from forming many bonds with Si.

\section{ACKNOWLEDGMENTS}

Calculations were performed using computational resources made available by the JREI under grant GR/M34454 and using the facilities of Oxford Supercomputing Centre. 


\section{REFERENCES}

[1] K. Maex, Materials Science and Engineering Reports 11, 53 (1993).

[2] M.-A. Nicolet and S. S. Lau in VLSI Electronics Microstructure Science, edited by N. G. Einspruch and G. B. Larrabee (Academic Press, New York, 1983), p 329.

[3] P. H. Giauque, Travail de Diplôme (Institut de Physique Expérimentale Université de Lausanne, Lausanne, 1992).

[4] H. L. Meyerheim, U. Döbler and A. Puschmann, Phys. Rev. B 44, 5738 (1991).

[5] K. Goto, J. Watanabe, T. Sukegawa, A. Fushida, T. Sakuma and T Sugii, Proceedings of IEEE $36^{\text {th }}$ International Reliability Physics Symposium, 363 (1998).

[6] K. Goto, A. Fushida, J. Watanabe, T. Sukegawa, Y. Tada, T. Nakamura, T. Yamazaki and T. Sugii, IEEE Transactions on Electronic Devices 46, 117 (1999).

[7] V. Scheuch, B. Voigtländer and H. P. Bonzel, Surf. Sci. 372, 71 (1997).

[8] H. J. W. Zandvliet, H. K. Louwsma, P. E. Hegeman and B. Poelsema, Phys. Rev. Lett. 75, 3890 (1995).

[9] W. S. Cho, J. Y. Kim, N. G. Park, I. W. Lyo, K. Jeong, S. S. Kim, D. S. Choi, C. N. Whang and K. H. Chae, Surf. Sci. 453, L309 (2000).

[10] W. Kohn and L.J. Sham, Phys. Rev. 140, A1133 (1965).

[11] S. D. Kenny, A. P. Horsfield and H. Fujitani, Phys. Rev. B, 62, 4899 (2000).

[12] S. Goedecker, M. Teter and J. Hutter, Phys. Rev. B 54, 1703 (1996).

[13] C.Hartwigsen, S. Goedecker and J. Hutter, Phys. Rev. B 58, 3641 (1998).

[14] The ABINIT code is a common project of the Universite Catholique de Louvain, Corning Incorporated, and other contributors (URL http://www.pcpm.ucl.ac.be/abinit).

[15] R. Stadler, W. Wolf, R. Podloucky, G. Kresse, J. Furthmüller and J. Hafner, Phys. Rev. 
B 54, 1729 (1996).

[16] S. Higai and T. Ohno, Appl. Surf. Sci. 166, 149 (2000)

[17] K. Miwa and A. Fukumoto, Phys. Rev. B 5214748 (1995).

[18] B. D. Yu, Y. Miyamoto, O. Sugino, T. Sasaki and T. Ohno, Phys. Rev. B 58, 3549 (1998).

[19] P. Zeppenfeld, M. Krzyzowski, C. Romainczyk, G. Comsa and M.G. Lagally, Phys. Rev. Lett. 72, 2737 (1994).

[20] P. Weakliem, Z. Zhang and H. Metiu, Surf. Sci. 336, 303 (1995).

[21] H. Yang, C. Zhu, J. Gao, Z. Xue and S. Pang 412-413, 236 (1998).

[22] A. natori and T. Kawabe, Surf. Sci. 433-435, 600 (1999).

[23] T. Kawabe and A. Natori, Surf. Sci. 462, 181 (2000).

[24] V. Milman, B. Winkler, J. A. White, C. J. Pickard, M. C. Payne, E. V. Akhmatskaya, R. H. Nobes, Int. J. Quant. Chem. 77, 895 (2000). The program CASTEP is developed and distributed by Molecular Simulations Inc., San Diego, CA, USA. See the Users Guide for details of the program.

[25] P. A. Bennett, D. G. Cahill and M. Copel, Phys. Rev. Lett. 73, 452 (1994). 


\section{TABLES}

TABLE I. The basis set for silicon is optimized to describe both the surface and the bulk. The variable adjusted during optimization is the cutoff radius $\left(r_{c}\right)$. where $r_{c}$ is given in Bohr radii. The bulk energy is measured relative to the converged plane wave result. The value of $r_{c}=8$ Bohr radii is chosen as an optimum value. Significant improvement on this requires the use of a triple numeric with double polarization (TNDP) basis set.

\begin{tabular}{lccccc}
\hline \hline basis & $\mathrm{a}_{0}(\AA)$ & $\mathrm{B}_{0}(\mathrm{GPa})$ & $\mathrm{U}_{\text {bulk }}(\mathrm{eV} /$ atom $)$ & $r_{\text {dimer }}(\AA)$ & $\mathrm{U}_{\text {surface }}(\mathrm{eV})$ \\
\hline DNP $\left(r_{c}=7\right)$ & 5.398 & 91.8 & 0.16 & 2.294 & 3.02 \\
DNP $\left(r_{c}=8\right)$ & 5.402 & 92.4 & 0.12 & 2.289 & 2.78 \\
DNP $\left(r_{c}=9\right)$ & 5.406 & 91.1 & 0.12 & 2.285 & 2.70 \\
TNDP $\left(r_{c}=9\right)$ & 5.389 & 93.4 & 0.03 & 2.270 & 2.70 \\
Plane waves & 5.383 & 94.1 & 0.00 & 2.265 & 2.65 \\
\hline \hline
\end{tabular}

TABLE II. The lattice constant and bulk modulus of $\mathrm{CoSi}_{2}$ found using various basis sets.

\begin{tabular}{lcc}
\hline \hline basis & $\mathrm{a}_{0}(\AA)$ & $\mathrm{B}_{0}(\mathrm{GPa})$ \\
\hline Minimal LCAO & 5.340 & 244 \\
DNP LCAO & 5.293 & 197 \\
Plane waves [15] & 5.283 & 200.2 \\
FLAPW [15] & 5.292 & 202 \\
\hline Experiment [3] & 5.365 & 171 \\
\hline \hline
\end{tabular}


TABLE III. The relative energies (in $\mathrm{eV}$ ) of the structures formed after the adsorption of a metal atom. The Co results are from this work. The number in parenthesis was evaluated using a well converged plane wave calculation. The $\mathrm{P}_{s}$ structure is taken as the zero of energy. Note that for DV structure, the missing Si atoms are included by using the chemical potential for bulk silicon. The results for $\mathrm{Ni}$ are calculated using the generalised gradient approximation.

\begin{tabular}{lccc}
\hline \hline Structure & Co & $\mathrm{Ti}[18]$ & $\mathrm{Ni}[16]$ \\
\hline $\mathrm{P}_{s}$ & 0.00 & 0.00 & 0.00 \\
$\mathrm{P}_{a}$ & 0.14 & & \\
$\mathrm{~T} 3$ & 0.49 & 0.25 & \\
$\mathrm{~T} 4$ & 1.07 & 0.52 & 0.31 \\
$\mathrm{H}$ & 0.11 & & 0.17 \\
$\mathrm{~L}$ & -0.24 & & 0.28 \\
$\mathrm{D}$ & 1.51 & & \\
$\mathrm{DV}$ & -1.86 & -0.82 & -0.19 \\
$\mathrm{U}_{D}$ & $-1.66)$ & -0.09 & -0.40 \\
$\mathrm{U}_{H}$ & -0.57 & & 0.19 \\
$\mathrm{U}_{L}$ & -0.59 & & \\
\hline \hline
\end{tabular}




\section{FIGURES}

FIG. 1. Seven sites are considered for Co adsorption on the $\mathrm{Si}(100)$ surface $\left(\mathrm{P}_{s}, \mathrm{P}_{a}\right.$, T3, T4, H, L, D). The eighth site (DV) corresponds to substituting a Co atom for a dimer. The remaining three sites $\left(\mathrm{U}_{D}, \mathrm{U}_{H}, \mathrm{U}_{L}\right)$ all lie beneath the surface.

FIG. 2. The relaxed geometries of the adsorbed Co atom at the seven surface sites $\left(\mathrm{P}_{s}, \mathrm{P}_{a}\right.$, T3,

$\mathrm{T} 4, \mathrm{H}, \mathrm{L}$, and $\mathrm{D})$, in the dimer vacancy site $(\mathrm{DV})$ and in the three sites below the surface $\left(\mathrm{U}_{D}\right.$, $\mathrm{U}_{H}$ and $\left.\mathrm{U}_{L}\right)$. 